\title{
Personalien
}

Todesfälle / Décès / Decessi

Alfred Lenzin (1940), † 1.6.2017,

Facharzt für Urologie, 3006 Bern

Peter Hiltbold (1924), † 14.7.2017,

Facharzt für Allgemeine Innere Medizin, 3600 Thun

Florin Enderlin (1926), † 22.7.2017,

Facharzt für Chirurgie, 4153 Reinach BL

Jürg Reichen (1946), † 25.7.2017,

3280 Murten

Andreas Benz (1942), † 5.8.2017,

Facharzt für Psychiatrie und Psychotherapie, 8032 Zürich

Wilhelm Meyer (1928), † 6.8.2017,

Facharzt für Anästhesiologie, 4500 Solothurn

Henrique Schenkel (1944), † 14.8.2017,

Facharzt für Psychiatrie und Psychotherapie, 3013 Bern

Parviz Farhoumand (1924), †15.08.17,

Spécialiste en médecine nucléaire et Spécialiste en radiologie, 1206 Genève

Praxiseröffnung /

Nouveaux cabinets médicaux /

Nuovi studi medici

GR

\section{Sabine Frei,}

Praktische Ärztin und Fachärztin für Allgemeine Innere Medizin, Hartbertstrasse 9, 7000 Chur

\section{VD}

\section{Gyana Antoni,}

Spécialiste en gynécologie et obstétrique, av. Alfred-Cortot 7E, 1260 Nyon

\section{AG}

Caroline Eva Alcantara Belisario,

Fachärztin für Allgemeine Innere Medizin,

FMH, Zentralstrasse 55a, 5610 Wohlen AG

\section{Aargauischer Ärzteverband}

Zur Aufnahme in den Aargauischen Ärzteverband haben sich als ordentlich praktizierende Mitglieder angemeldet:

Susanne Bock, 4053 Basel, Fachärztin für Allgemeine Innere Medizin, FMH, Praxiseröffnung in Praxisgemeinschaft in Rheinfelden seit 14. August 2017

Corinna Kuhn, 8966 Oberwil-Lieli, Fachärztin für Allgemeine Innere Medizin, FMH, Praxiseröffnung in Praxisgemeinschaft in Jonen seit 1. April 2017

Susanne Vögeli, 8906 Bonstetten, Fachärztin für Ophthalmologie, angestellt in Praxis in Wettingen seit 1. Juli 2017

Als Chef- und Leitender Arzt hat sich angemeldet:

Stefan Grunder, 3205 Gümmenen, Facharzt für Allgemeine Innere Medizin, FMH, Medizinischer Direktor in der Hirslanden Klinik Aarau seit 1. Mai 2017

Diese Kandidaturen werden in Anwendung von Art. 5 der Statuten des Aargauischen Ärzteverbandes veröffentlicht. Einsprachen müssen innert 14 Tagen seit der Bekanntmachung schriftlich und begründet der Geschäftsleitung des Aargauischen Ärzteverbandes eingereicht werden. Nach Ablauf der Einsprachefrist entscheidet die Geschäftsleitung über Gesuch und allfällige Einsprachen.

\section{Ärztegesellschaft des Kantons Bern} Ärztlicher Bezirksverein Bern Regio

Zur Aufnahme als ordentliches Mitglied hat sich angemeldet:

Fabienne Ruchti, Fachärztin für Allgemeine Innere Medizin, FMH, Monbijoustrasse 68, 3007 Bern

Einsprachen gegen diese Vorhaben müssen innerhalb 14 Tagen seit der Veröffentlichung schriftlich und begründet beim Präsidenten des Ärztlichen Bezirksvereins Bern Regio eingereicht werden. Nach Ablauf der Frist entscheidet der Vorstand über die Aufnahme der Gesuche und über die allfälligen Einsprachen.

\section{Ärztegesellschaft des Kantons Luzern}

Zur Aufnahme in unsere Gesellschaft Sektion Stadt hat sich gemeldet:

Irene Wenaweser-Gander, Fachärztin für Ophthalmologie, FMH, Augenarztpraxis Ladengasse, Zentralstrasse 16, 6030 Ebikon

Einsprachen sind innert 20 Tagen nach der Publikation schriftlich und begründet zu richten an: Ärztegesellschaft des Kantons Luzern, Schwanenplatz 7, 6004 Luzern

Ärztegesellschaft des Kantons Schwyz

Zur Aufnahme in die Ärztegesellschaft des Kantons Schwyz hat sich angemeldet:

Dai-Do Do, Facharzt für Angiologie und Allgemeine Innere Medizin, FMH, Cardiance Clinic in Pfäffikon

Einsprachen gegen diese Aufnahme richten Sie schriftlich innert 20 Tagen an Dr. med. Uta Kliesch, Maria-Hilf-Strasse 9, 6430 Schwyz oder per Mail an uta.kliesch@hin.ch

\section{Ärztegesellschaft Thurgau}

Zum Eintritt in die Ärztegesellschaft Thurgau haben sich gemeldet:

Nicoletta Birgit Denize, Reichenaustrasse 36 D-78467 Konstanz, Fachärztin für Dermatologie und Venerologie

Barbara Erdélyi-Canavese, Thundorferstrasse 120, 8500 Frauenfeld, Fachärztin für Neurologie

Barbara Peter Hauser, Stählistrasse 3, 8280 Kreuzlingen, Fachärztin für Chirurgie und Traumatologie FMH 